\title{
Meaning in life and well-being of older stroke survivors in Chinese communities: Mediating effects of mastery and self-esteem
}

\author{
Jingjin Shao ${ }^{1 *}$, Jiliang Shen ${ }^{2}$, Qinghua Zhang ${ }^{3}$, Tian Lin ${ }^{4}$ \\ ${ }^{1}$ Research Center of Mental Health Education and Faculty of Psychology, Southwest University, Chongqing, China; \\ *Corresponding Author: jingjinshao@gmail.com \\ ${ }^{2}$ Institute of Developmental Psychology, Beijing Normal University, Beijing, China \\ ${ }^{3}$ Department of Public Administration, Chongqing Youth Vocational and Technical College, Chongqing, China \\ ${ }^{4}$ Department of Psychology, University of Florida, Gainesville, USA
}

Received 11 February 2013; revised 20 March 2013; accepted 5 April 2013

Copyright (C) 2013 Jingjin Shao et al. This is an open access article distributed under the Creative Commons Attribution License, which permits unrestricted use, distribution, and reproduction in any medium, provided the original work is properly cited.

\begin{abstract}
The study aims to examine the role of mastery and self-esteem as a potential mediator of the relationship of meaning in life and well-being among older Chinese stroke survivors. A crosssectional study was conducted in 214 community-dwelling older stroke survivors (128 men and 86 women), ranging from 60 years to 88 years old. The meaning in life, mastery, selfesteem and subjective well-being were measured. The results indicated that: 1) The meaning in life and subjective well-being of stroke survivors were significantly positive correlated; 2) Mastery and self-esteem played partial mediating roles between existential vacuum and subjective well-being; Self-esteem played full a mediating role between suffer acceptance and subjective well-being; Mastery played a full mediating role between life control and subjective well-being; Mastery played a partial mediating role between death acceptance and subjective well-being; Self-esteem also played a partial mediating role between mastery and subjective wellbeing.
\end{abstract}

Keywords: Stroke; Meaning in Life; Mastery; Self-Esteem; Well-Being

\section{INTRODUCTION}

Strokes generally result in serious and debilitating chronic health problems and they mostly occur later in life [1,2]. China has approximately 7.5 million stroke patients, with more than 2.5 million new cases and 1.6 million deaths annually [3]. Combined with the declining stroke mortality trends, High disability rate caused by stroke have become increasing prominent, about threefourths patients have varying degrees of disability [4]. The associated residual impairments and disabilities significantly limit the well-being of older adults [1]. However, the resulting physical disabilities are not necessarily correlated with well-being. Some people manage to retain their sense of well-being despite their stroke-related disabilities and impairments, whereas others demonstrate significant emotional and interpersonal decline [1,5]. Yet, few studies have examined the well-being of older adult stroke survivors in Chinese communities [6], particularly in maintaining or restoring their well-being following stroke [7].

Meaning in life refers to making sense, order or coherence out of one's existence and seeking for valuable goals to be achieved and fulfillment [8]. The important role of meaning in life played in trauma (including disease) treatment has received wide attention [8,9], which is "unique and irreplaceable in the case of personal crisis and major setback" [10]. Those individuals who can resolve the crisis and establish meaning in life are more adaptable and report less stress and greater well-being [11]. Thus, meaning in life is a critical element to maintain psychological well-being [12-14]. Empirical studies have examined the relationship between meaning in life and well-being in chronic disease, yet little attention has been paid to the underlying mechanism that promotes or maintains well-being $[15,16]$.

The sense of mastery is beliefs of one's that they personally can control or regain events and their life [17], which is expected to be able to mitigate the stress and 
depressions of cancer patients [18]. Self-esteem is the overall positive evaluation of self and is significantly associated with well-being of stroke patients $[19,20]$. The meanings in life, sense of mastery and self-esteem have attracted long time attentions [21]. Both cognitive adaptation theories [17] and shattered assumptions theory [22] emphasized the positive role of meaning in life, mastery and self-esteem in trauma response and has been confirmed by many studies on disease response [23,24]. The sudden attack of stroke may cause body out of control, such as hemiplegia, sensory loss, hemispatial neglect and aphasia. The patients also face problems of continuing long-term treatment and being dependent on care. Feeling out of control and sense of uncertainty will become a major psychological experience of stroke survivors. The crisis phenomenon that emerges after stroke is metaphorically depicted as "a struggle in the darkness" in a "boundary situation", where the issues touch on life and death, fate and future, and meaning and meaninglessness [25]. so that regaining control over body and living and self-esteem are important task for stroke survivors [17, 26]. Therefore, it can be assumed that there is a strong positive correlation between meaning in life and subjective well-being. The mastery and self-esteem may play a mediating role between them.

\section{METHODS}

\subsection{Participants}

In this descriptive cross-sectional correlation study, all participants were initially identified from the hospital records of eight Community Health Service centers in China. This study was approved by the Institutional Review Boards of Beijing Normal University. Inclusion criteria are as follows: 1) diagnosis via head CT or MRI; 2) age 60 years and older and suffering from the disease for more than four weeks; 3) absence of obvious aphasia and adequate cognitive function to participate in the study; 4) absence of severe post-stroke depression; 5) absence of previous psychiatric history; and 6) informed consent was sought by telephone from the subjects or their relatives.

A total of 232 older community-dwelling stroke survivors participated in this study. Eighteen participants were excluded from the analyses because they had incomplete variables of interest because of fatigue. The final sample consisted of 214 elderly stroke survivors (128 men; 86 women), with a mean age of 70.29 years ( $S D=7.64$, range 60 years to 88 years). The average survival after their first stroke was 7.66 years $(S D=$ 6.20). Stroke was classified into five types: a) cerebral thrombosis (30.8\%); b) cerebral infarction (57.9\%); c) cerebral hemorrhage (9.8\%); d) subarachnoid hemorrhage $(0.5 \%)$; and e) undifferentiated type $(0.9 \%)$. Up to
$78 \%$ percent of the participants had a partner (i.e., legal spouse or common law spouse). Prior to retirement, 27.6\% of the subjects were farmers or unemployed, $23.4 \%$ were merchants, $17.8 \%$ were technician, $20.6 \%$ were middle managers, and $10.7 \%$ were senior managerial staff. The average number of years of education was $6.56(S D=$ $4.65)$, and the average number of co-morbidities was $2.65(S D=1.65)$.

\subsection{Data Collection Procedures}

The data used in this study were collected from July 2010 to December 2010 in Mainland China. Eight Community Health Service centers helped identify the participant pool by reviewing patient files. Pre-screening was performed by key contact personnel from the health service centers using Mini Mental Status Examinations and Self-Rating Depression.

After confirming study eligibility by telephone, appointments were scheduled to obtain informed consent and to conduct the one-time face-to-face interview with older adult stroke survivors at a convenient date, time, and location. The investigation was conducted by primary investigators, and most of the participants were interviewed at home or in health service center offices to ensure privacy. All participants completed a multi-section questionnaire, including brief demographics, disease survey, and self-report measures of meaning in life, mastery, self-esteem and subjective well-being, during the one-on-one meeting with a trained investigator. For participants who could not read or write but agreed to participate, the questionnaire was read aloud and the participants' answers were recorded using a digital recording pen. The average time required to complete the questionnaires was $50 \mathrm{~min}$.

\subsection{Measures}

\subsubsection{Meaning in Life}

Meaning in life was assessed using the Meaning in Life Scale developed by Wu [27] and adapted by Shao [28]. It is a multi-dimensional measuring tool based on the Frankl's theory of logotherapy and the Life Attitude Profile [29]. This scale consists of six dimensions: will to meaning, existential vacuum, life purpose, life control, suffer acceptance, and Death acceptance. It comprises 18 items with a score ranging from 1 ("strongly disagree") to 5 ("strongly agree"), among which existential vacuum was scored reversely. The coefficients of internal consistency of the total measure and six factors were 0.88 , $0.68,0.80,0.79,0.75,0.67$, and 0.48 , respectively.

\subsubsection{Self-Mastery Scale}

The Self-Mastery Scale (SMS) developed by Pearlin and Schooler [30] was used to measure the extent to 
which one regards one's life-chances as being under one's own control in contrast to being fatalistically ruled. Respondents described their feelings about 7 items such as "My future mostly depends on me" on a 4-point Likert scale ranging from 1 (strongly disagree) to 4 (strongly agree). Responses to negatively worded items were reverse-coded, and the total score ranged from 7 to 28 . The Cronbach's $\alpha$ was 0.89 in this study.

\subsubsection{Self-Esteem Scale}

The Self-Esteem Scale (SES) developed by Rosenberg [31] was used to measure overall feeling of self value and self acceptance. It was a 10-item scale employing a 4-point Likert-type format, ranging from 1 (strongly disagree) to 4 (strongly agree). Items 3, 5, 9 and 10 were scored reversely. The total scores were in the range of 10 40. The Cronbach's $\alpha$ was 0.90 in this study.

\subsubsection{Subjective Well-Being}

The subjective well-being questionnaire developed by Diener [32] was used in this study. It consists of three sub-scales of life satisfaction (5 items), positive affect frequency (6 items), and passive affect frequency (8 items). The items in life satisfaction ranged from 1 (strongly disapprove) to 7 (strongly approve). Items in the positive and passive emotion frequency refer to the emotions experienced within the latest week, ranging from 1 (have not yet experienced) to 7 (experience it all the time). Items in the passive emotion frequency were scored reversely, and the total subjective well-being score was calculated. Cronbach's $\alpha=0.92$ in this study.

\subsection{Data Analysis}

Covariance structure modeling was used to examine the relationship between meaning in life, mastery, selfesteem and subjective well-being. An important feature of the analysis is the ability to verify multiple pathways and multiple variables simultaneously. The hypothesized model was tested via maximum likelihood estimation using Amos 7.0. The following indices were used to evaluate the overall model fit: the $X^{2}$ goodness-of-fit statistic, the goodness-of-fit index $(G F I)$, the non-normed fit index $(N N F I)$, the incremental fit index $(I F I)$, the Tucker-Lewis index (TLI), the comparative fit index ( $C F I$ ), and the root mean square error of approximation (RMSEA) [33]. In addition, the data conformed to SEM assumption conditions and theoretical hypothesis when considering model fit. Patient characteristics (sociodemographics, disease, and treatment variables) were selected and included in the model as controls if they were significantly correlated $(p<0.05)$ with subjective wellbeing.

\section{RESULTS}

\subsection{Relationships among Meaning in Life, Mastery, Self-Esteem and Subjective Well-Being}

The descriptive analysis and Pearson correlation analysis on meaning in life, mastery, self-esteem and subjective well-being were given in Tables 1 and 2 respectively. The results showed that meaning in life, astery, self-

Table 1. Means and standard deviations $(\mathrm{N}=214)$.

\begin{tabular}{cccccccccc}
\hline & WM & EV & LP & LC & SA & DA & MS & SE & SWB \\
\hline$M$ & 3.84 & 3.89 & 3.60 & 3.96 & 4.12 & 4.12 & 20.08 & 31.55 & 95.95 \\
$S D$ & 0.81 & 0.98 & 0.86 & 0.79 & 0.70 & 0.69 & 4.83 & 6.01 & 17.87 \\
\hline
\end{tabular}

Notes: $\mathrm{WM}=$ will to meaning; $\mathrm{EV}=$ existential vacuum; $\mathrm{LP}=$ life purpose; $\mathrm{LC}=$ life control; $\mathrm{SA}=$ suffer acceptance; $\mathrm{DA}=$ death acceptance; $\mathrm{MS}=$ Mastery; $\mathrm{SE}=$ Self-esteem; $\mathrm{SWB}=$ subjective well-being.

Table 2. Correlation matrix $(\mathrm{N}=214)$.

\begin{tabular}{|c|c|c|c|c|c|c|c|c|}
\hline & 1 & 2 & 3 & 4 & 5 & 6 & 7 & 8 \\
\hline 1. WM & 1 & & & & & & & \\
\hline 2. $\mathrm{EV}$ & $0.36^{* *}$ & 1 & & & & & & \\
\hline 3. LP & $0.61^{* *}$ & $0.60^{* *}$ & 1 & & & & & \\
\hline 4. LC & $0.50^{* *}$ & $0.68^{* *}$ & $0.62^{* *}$ & 1 & & & & \\
\hline 5. SA & $0.38^{* *}$ & $0.35^{* *}$ & $0.42^{* *}$ & $0.42^{* *}$ & 1 & & & \\
\hline 6. DA & $0.28^{* *}$ & $0.23^{* *}$ & $0.20^{* *}$ & $0.34^{* *}$ & $0.14^{* *}$ & 1 & & \\
\hline 7. MS & $0.43^{* *}$ & $0.71^{* *}$ & $0.59^{* *}$ & $0.79^{* *}$ & $0.34^{* *}$ & $0.38^{* *}$ & 1 & \\
\hline 8. SE & $0.40^{* *}$ & $0.70^{* *}$ & $0.61^{* *}$ & $0.66^{* *}$ & $0.44^{* *}$ & $0.22^{* *}$ & $0.70^{* *}$ & 1 \\
\hline 9. SWB & $0.39^{* *}$ & $0.72^{* *}$ & $0.56^{* *}$ & $0.68^{* *}$ & $0.41^{* *}$ & $0.34^{* *}$ & $0.74^{* *}$ & $0.73^{* *}$ \\
\hline
\end{tabular}

Notes: ${ }^{*} p<0.05 ;{ }^{* *} p<0.01 ;{ }^{* * *} p<0.001$. 
esteem and subjective well-being of stroke patients were significantly and positively correlated with each other ( $p$ $<0.01$ ). These results reveal that all the preconditions for mediation identified by Baron and Kenny were satisfied [34].

\subsection{Mediating Effects}

The mediating effects of mastery and self-esteem were examined by structural equation modeling (AMOS 7.0). The model was calibrated based on modified index and theoretical analysis. The model yielded satisfactory fit indices, $X^{2} / d f=1.546\left(X^{2}=17.011, d f=11\right), G F I=$ 0.983, NFI $=0.985, I F I=0.995, T L I=0.983, C F I=$ $0.995, R M S E A=0.051$. The results showed that the model fitted well.

It can be seen from Figure 1 that the existential vacuum can directly impact subjective well-being $(\beta=0.27$, $p<0.001)$ and can also impact subjective well-being through mastery $(\beta=0.33, p<0.001)$ and self-esteem $(\beta$ $=0.37, p<0.001)$. The mediating effects accounted for 47.7\%; Life control can impact subjective well-being through mastery $(\beta=0.53, p<0.001)$ and mastery played a full mediation role; Suffer acceptance can impact subjective well-being through self-esteem $(\beta=0.18$, $p<0.001$ ) and self-esteem played a full mediation role; Death acceptance can impact subjective well-being directly $(\beta=0.10, p<0.05)$ and can also impact subjective well-being though mastery $(\beta=0.12, p<0.01)$. The mastery played a partial mediation role, which accounting for $32.7 \%$ of total mediating effects. In addition, mastery can directly impact subjective well-being ( $\beta=$ $0.30, p<0.001$ ) and can also impact subjective wellbeing through self-esteem $(\beta=0.38, p<0.001)$. The mediating effect accounted for $27.8 \%$ of total effects.

\section{DISCUSSION}

With the rising of positive psychology, the relationships between meaning in life and well-being have received great attention from psychologists but the underlying mechanism of meaning in life has always been ignored $[15,16]$. The relationships between meaning in life and subjective well-being of stroke patients and its potential mechanism were revealed in this study by structural equation modeling. It was found that the meaning in life and subjective well-being of stroke patients were significantly and positively correlated, which was consistent with the results of relevant researches on adjustment to chronic diseases [12-14]. This indicated that meaning in life was an essential element and precondition to subjective well-being of older stroke survivors in Chinese communities.

How does meaning in life positively impact subjective well-being? This study showed that mastery and selfesteem played different degree of meditating roles between meaning in life and subjective well-being: 1) direct effects: existential vacuum and Death acceptance had a positive prediction function on subjective wellbeing; 2) Mediating effects of mastery: existential vacuum, life control and acceptance can impact subjective well-being through mastery; 3) Mediating effects of self-

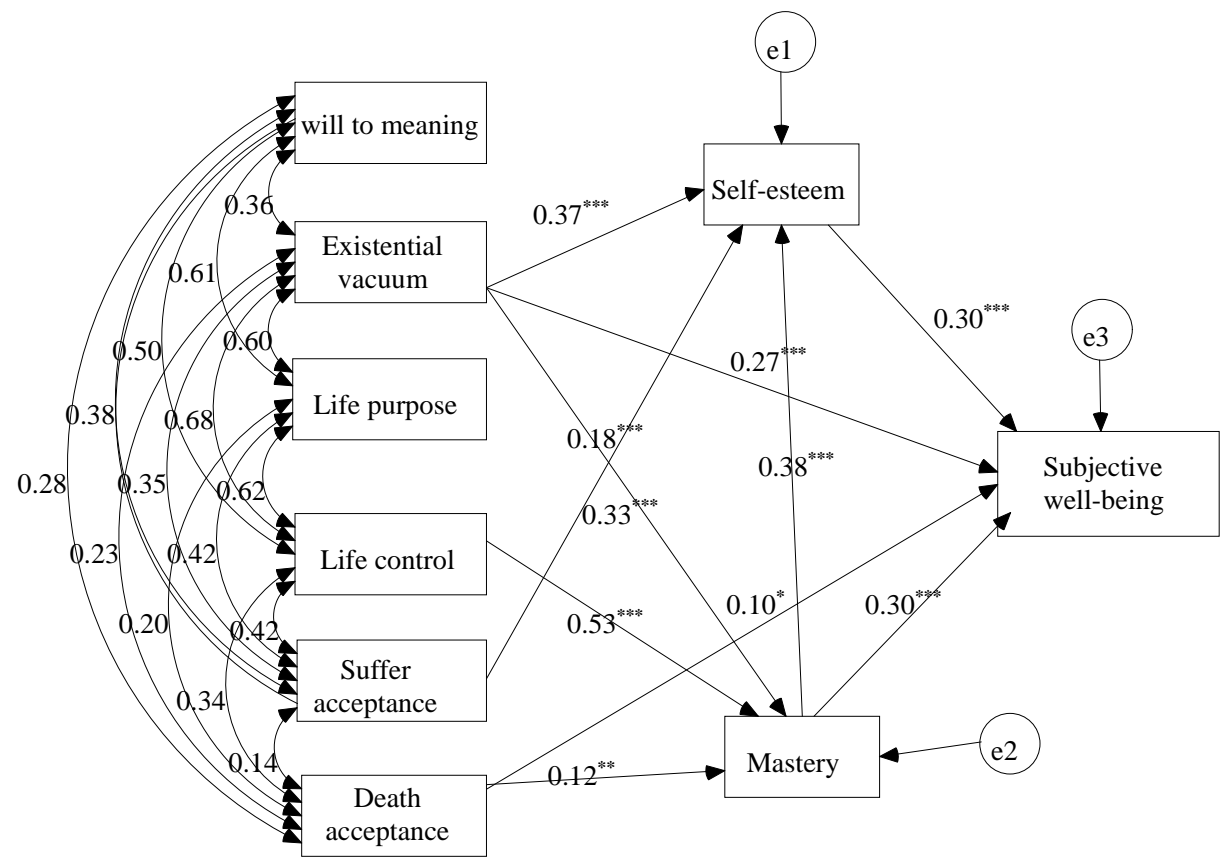

Figure 1. Path analysis on meaning in life, mastery, self-esteem and subjective well-being; Note: standardized regression coefficients ${ }^{*} p<0.05 ;{ }^{* *} p<0.01 ;{ }^{* * *} p<0.001$. 
esteem: existential vacuum and suffer acceptance impacted subjective well-being through self-esteem. Different dimensions of meaning in life had different relationships with subjective well-being and different mechanisms: will to meaning and life purpose did not have significant influence on subjective well-being. When studying the impacts of existential vacuum, life control, suffer acceptance and Death acceptance on subjective well-being, the different roles of mastery and self-esteem played in pathways needed to be considered. This indicated that the interrelationship between meaning in life and subjective well-being were affected by the specific dimension of meaning in life. In addition, self-esteem also was the mediator in the pathway from mastery to subjective well-being. This result was consistent with Forbes and Fife that the mastery patients gained improved their self-worth, thus contributing to increase well-being [23,24].

The relationships between meaning in life and subjective well-being can be explianed by cognitive adaptation theory and shattered assumptions. People have a strong basic assumption that can control their life, the surroundings and events happened to them. The positive factors founded from traumatic events can help patients regain cognitive consistency [22] so that the patients can discover the meaning and value in life from the helplessness traumatic experience and gain a sense of control of the current situation and enhance self-esteem [17], thus increase the patients' sense of subjective well-being. It needs to be noted that mastery in the situation of serious disease is not necessarily the actual physical control. Creation of positive illusion to seek for changes of meaning may also be helpful to regain mastery and efficacy against disease events and enhance self-esteem, thereby contributing to successful adjustment and adaptation of stroke events.

Several limitations should be considered when evaluating the findings from this study. First, the cross-sectional design limits our ability to define the causal relationship between meaning in life, mastery, self-esteem and well-being, which is required in future prospective studies. Second, convenient and "snowball" sampling methods were mainly used in this study. Most of the participants were interviewed in their homes, considering many patients have a restrictive physical disability, hemiplegia, aphasia, and so on. Therefore, large-scale sampling is impossible, which may limit the generalizability of the research results. Third, although the quantitative research adopted in this study helped scientifically describe and define the relationships between variables, every stroke patient's adjustment is a special "life story". Different disease experiences affect their understanding of meaning in life. Therefore, qualitative research provides a detailed practically reasonable basis for quantita- tive studies. Quantitative research and qualitative research complement each other, allowing a more intensive study and providing complete understanding of the stroke experience [7].

\section{CONCLUSIONS}

1) The meaning in life and subjective well-being of stroke patients were significantly and positively correlated.

2) The subjective well-being was affected by existential vacuum, life control, suffer acceptance and Death acceptance through the mediating effects of mastery and self-esteem. Mastery and self-esteem played a partial mediating role between existential vacuum and subjective well-being; self-esteem played a full mediating role between suffer acceptance and subjective well-being; mastery played a full mediating role role between life control and subjective well-being; mastery played a partial mediating role role between Death acceptance and subjective well-being; self-esteem played a partial mediating role between mastery and subjective well-being.

\section{ACKNOWLEDGEMENTS}

This study was supported by the Southwest University for researcher groups (TR201204-7), the Social Sciences Foundation of the Ministry of Education in China (08JJDXLX268) and the 211 Project for SWU (NSKD11055).

\section{REFERENCES}

[1] Clarke, P.J. and Black, S.E. (2005) Quality of life following stroke: Negotiating disability, identity, and resources. Journal of Applied Gerontology, 24, 319-336. doi:10.1177/0733464805277976

[2] Wang, Y.J. (2004) Stroke unit. Scientific and Technological Literature Publishing House, Beijing.

[3] Yin, L. (2008) 2007 year book of health in the People's Republic of China. People's Medical Publishing House, Beijing.

[4] Su, D.F. (2008) Stroke prevention from perspective of basic research. Chinese Journal of Geriatric Heart Brain and Vessel Diseases, 10, 86-87.

[5] Wilson, I.B. and Cleary, P.D. (1995) Linking clinical variables with health-related quality of life: A conceptual model of patient outcomes. Journal of the American Medical Association, 273, 59-65. doi:10.1001/jama.1995.03520250075037

[6] Shao, J.J., Shen, J.L., Lin, T., et al. (2010) Relationship between compliance and quality of life of senior survivors of stroke in the community. Chinese Journal of Clinical Psychology, 18, 622-625.

[7] Clarke, P. (2009) Understanding the experience of stroke: A mixed-method research ageda. The Gerontologist, 49, 293-302. doi:10.1093/geront/gnp047 
[8] Reker, G.T., Peacock, E.J. and Wong, E.T. (1987) Meaning and purpose in life and well-being: A life-span perspective. Journal of Gerontology, 42, 44-49. doi:10.1093/geronj/42.1.44

[9] Frankl, V.E. (1963) Man’s search for meaning: An introduction to logotherapy. Pocket Books, New York.

[10] Li, H. (2006) Self-transcendence meaning of life moderates in the relation between college stress and psychological well-being. Acta Psychological Sinica, 38, 422427.

[11] Zika, S. and Chamberlain, K. (1992) On the relation between meaning in life and subjective well-being. British Journal of Psychology, 83, 133-145. doi:10.1111/j.2044-8295.1992.tb02429.x

[12] Affleck, G., Tennen, H., Croog, S., et al. (1987) Causal attribution, perceived benefits, and morbidity after a heart attack: An 8-year study. Journal of Consulting and Clinical Psychology, 55, 29-35. doi:10.1037/0022-006X.55.1.29

[13] Jim, H.S. and Andersen, B.L. (2007) Meaning in life mediates the relationship between social and physical functioning and distress in cancer survivors. British Journal of Health Psychology, 12, 363-381. doi:10.1348/135910706X128278

[14] Thomoson, N.J., Coker, J., Krause, J. and Henry, E. (2003) Purpose in life as a mediator of adjustment after Spinal Cord Injury. Rehabilitation Psychology, 48, 100-108. doi:10.1037/0090-5550.48.2.100

[15] Ho, M.Y., Cheung, F.M. and Cheung, S.F. (2010) The role of meaning in life and optimism in promoting wellbeing. Personality and Individual Differences, 48, 658663. doi:10.1016/j.paid.2010.01.008

[16] Zhang, S.Y., Xu, Y. and Yang, H.K. (2010) The connotation, measurement and function of meaning in life. $A d$ vances in Psychological Science, 18, 1756-1761.

[17] Taylor, S.E. (1983) Adjustment to threatening events: Theory of cognitive adaptation. American Psychologist, 38, 1161-1173. doi:10.1037/0003-066X.38.11.1161

[18] Champion, V., Williams, S.D., Miller, A., et al. (2007) Quality of life in long-term survivors of ovarian germ cell tumors: A gynecologic oncology group study. Gynecologic Oncology, 105, 687-694. doi:10.1016/j.ygyno.2007.01.042

[19] Fung, L.C.L., Liu, M.L. and Chau, J.P. (2006) Relationship between self-esteem and the occurrence of depression following a stroke. Journal of Clinical Nursing, 15, 505-506. doi:10.1111/j.1365-2702.2006.01328.x

[20] Keppel, C.C. and Crowe, S.F. (2000) Changes to body image and self-esteem following stroke in young adults. Neuropsychological Rehabilitation, 10, 15-31. doi:10.1080/096020100389273
[21] Das, A.K. (1998) Frankl and the realm of meaning. Journal of Humanistic Education and Development, 36, 199- 211. doi:10.1002/j.2164-4683.1998.tb00392.x

[22] Janoff-Bulman, R. (1992) Shattered assumptions: Towards a new psychology of trauma. Free Press, New York.

[23] Forbes, D.A. (2001) Enhancing mastery and sense of coherence important determinants of health in older adults. Geriatric Nursing, 22, 29-32. doi:10.1067/mgn.2001.113532

[24] Fife, B.L. (2005) The role of constructed meaning in adaptation to the onset of life-threatening illness. Social Science and Medicine, 61, 2132-2143. doi:10.1016/j.socscimed.2005.04.026

[25] Nilsson, L., Jansson, L. and Norberg, A. (1999) Crisis phenomena after stoke reflected in an existential perspective. International Journal of Aging and Human Development, 49, 259-277. doi:10.2190/UUAE-TQ52-LUQ3-JVLR

[26] Ellis-Hill, C.S., Payne, S. and Ward, C. (2000) Self-body split: Issues of identity in physical recovery following a stroke. Disability and Rehabilitation, 22, 725-733. doi:10.1080/09638280050191990

[27] Wu, Y.S. (2009) Development of meaning in life scale for terminal cancer patients. Master Thesis, Guangxi Medical University, Nanning.

[28] Shao, J.J. (2011) The relationship between meaning in life and well-being in older adult stroke survivors. Ph.D. Thesis, Beijing Normal University, Beijing.

[29] Ho, Y.C. (1990) The life attitude profile: A study of reliability and validity. Bulletin of National Taiwan Normal University, 35, 71-94.

[30] Pearlin, L.I. and Schooler, C. (1978) The structure of coping. Journal of Health and Social Behavior, 24, 2-15. doi:10.2307/2136319

[31] Rosenberg, M. (1965) Self-esteem scale. In: Wang, X.D. and Wang, X.L., Eds., Rating Scales for Mental Health, Chinese Mental Health Journal, Beijing, 318-320.

[32] Diener, E., Diener, M. and Diener, C. (1995) Factors predicting the subjective well-being of nations. Journal of Personality and Social Psychology, 68, 653-663. doi:10.1037/0022-3514.68.4.653

[33] Hau, K.T., Wen, Z.L. and Chen, Z.J. (2004) Structural equation model and its applications. Educational Science Publishing House, Beijing.

[34] Baron, R.M. and Kenny, D.A. (1986) The moderatormediator variable distinction in social psychological research: Conceptual, strategic, and statistical considerations. Journal of Personality and Social Psychology, 51, 1173-1182. doi:10.1037/0022-3514.51.6.1173 